\title{
Numerical modelling of diesel spray using the Eulerian multiphase approach
}

\author{
Milan Vujanovićc ${ }^{\mathrm{a}}$, Zvonimir Petranovićc ${ }^{\mathrm{a}, *}$, Wilfried Edelbauer ${ }^{\mathrm{b}}$, Jakov Baleta ${ }^{\mathrm{a}}$, Neven Duić ${ }^{\mathrm{a}}$ \\ ${ }^{a}$ Faculty of Mechanical Engineering and Naval Architecture, University of Zagreb, Croatia \\ ${ }^{\mathrm{b}}$ AVL List GmbH, Graz, Austria
}

\section{A R T I C L E I N F O}

\section{Article history:}

Available online $\mathrm{xxxx}$

\section{Keywords:}

Diesel engine

Spray process

Eulerian framework

Numerical modelling

\begin{abstract}
A B S T R A C T
This research investigates high pressure diesel fuel injection into the combustion chamber by performing computational simulations using the Euler-Eulerian multiphase approach. Six diesel-like conditions were simulated for which the liquid fuel jet was injected into a pressurised inert environment $\left(100 \% \mathrm{~N}_{2}\right)$ through a $205 \mu \mathrm{m}$ nozzle hole. The analysis was focused on the liquid jet and vapour penetration, describing spatial and temporal spray evolution. For this purpose, an Eulerian multiphase model was implemented, variations of the sub-model coefficients were performed, and their impact on the spray formation was investigated. The final set of sub-model coefficients was applied to all operating points. Several simulations of high pressure diesel injections (50,80, and $120 \mathrm{MPa}$ ) combined with different chamber pressures (5.4 and 7.2 MPa) were carried out and results were compared to the experimental data. The predicted results share a similar spray cloud shape for all conditions with the different vapour and liquid penetration length. The liquid penetration is shortened with the increase in chamber pressure, whilst the vapour penetration is more pronounced by elevating the injection pressure. Finally, the results showed good agreement when compared to the measured data, and yielded the correct trends for both the liquid and vapour penetrations under different operating conditions.
\end{abstract}

(c) 2015 Elsevier Ltd. All rights reserved.

\section{Introduction}

Diesel engines produce pollutant emissions that cause environmental problems and can be harmful to human health. Allowable pollutant emissions from diesel engines have been regulated over the last few decades and new, more stringent regulations are expected within the next years. These regulations include the European emission standards arising from one of the governmental policies as an option for accomplishing cleaner production [1]. Due to the promotion of biofuels by the European Union [2], diesel engines must be subject to further development and meet higher efficiency standards [3] in order to remain the most used transportation vehicle powering system on the market. A significant amount of $\mathrm{CO}_{2}$ is released into the atmosphere by combusting fossil fuels [4], and a rapid emission reduction (up to 85\%) has to be achieved by 2050 [5]. It is reasonable to use the diesel engines as the internal combustion engine power source due to their more efficient energy conversion and higher safety factor when

* Corresponding author at: Ivana Lučića 5, 10002 Zagreb, Croatia. Tel.: +385 1 6168494.

E-mail address: zvonimir.petranovic@fsb.hr (Z. Petranović). compared to the spark ignition engines [6]. In internal combustion diesel engines there is mostly diffusion combustion present, meaning that the spray characteristics have the direct influence on the fuel energy conversion and the formation of harmful substances [7-11]. There are challenges associated with having a very short amount of time available for the fuel spray to atomise and form an adequate mixture for quality combustion. Therefore, suitable fuel injectors are needed to provide sufficient control on the spray process and to meet the basic requirements for the atomisation and mixing process. High pressure injectors are one of the most commonly used injectors in commercial applications today [12]. They are designed to improve the atomisation process and to increase the turbulence levels within the combustion chamber for better mixing between the air and fuel. Numerous studies about spray processes have helped engineers to establish the criteria needed for the designing and developing more efficient combustion devices, whilst minimising the pollutant emissions $[10,13,14]$. The understanding of the complex nature of the fuel spray formed by high pressure injectors in experimental investigations is limited and this understanding can be significantly improved by numerical simulations. It can be stated, that the uncertainties arising from the experiments can be figured out by performing numerical 


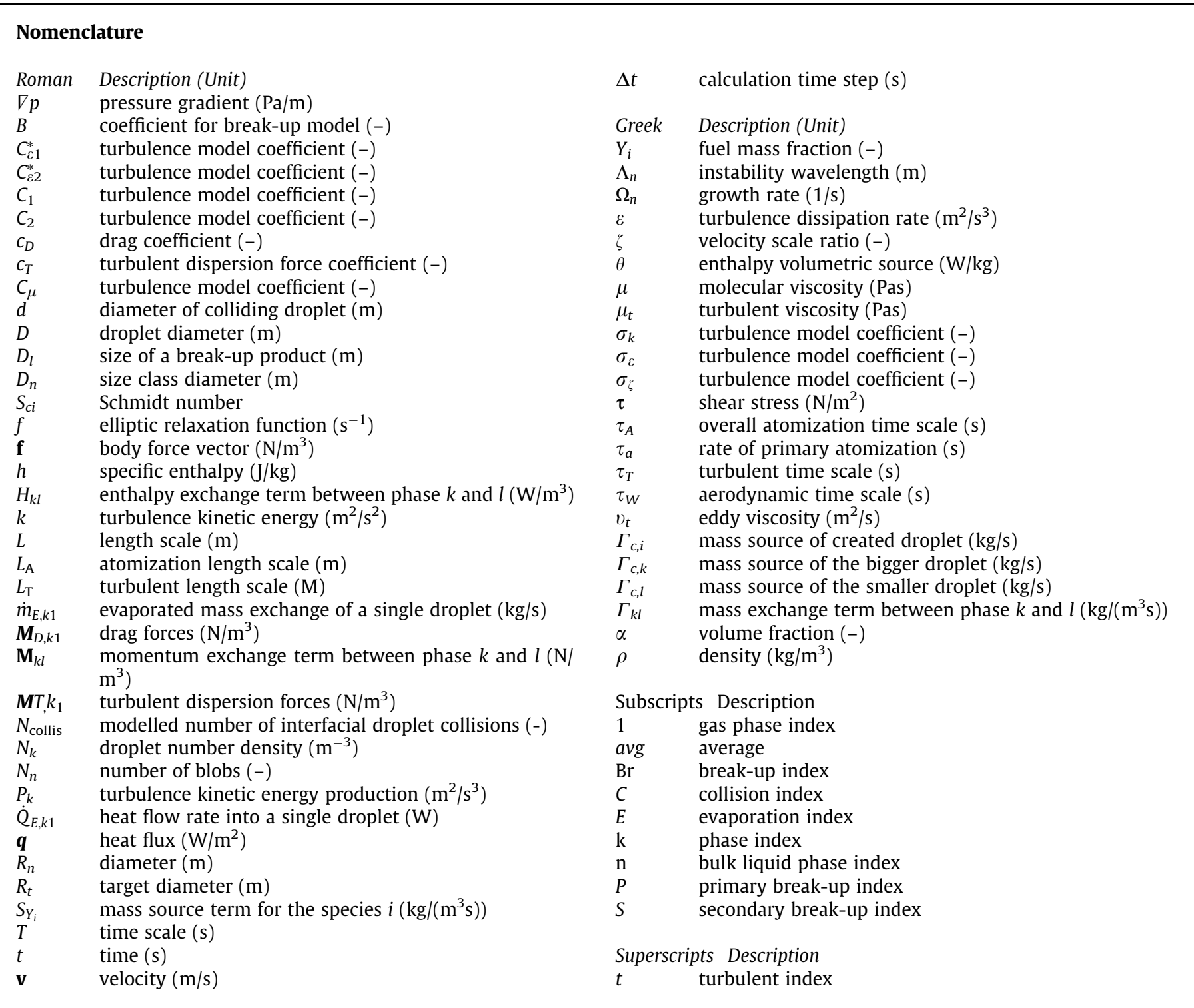

simulations [15]. Numerical modelling of spray processes is a very challenging task compared to a single phase flow. The challenges arise due to the fluid interfaces between the phases and the property variations across these interfaces. Thus, the spray models demand complicated techniques for coupling the dynamics of the liquid droplets and the gas carrier. A variety of strategies have been formulated over past years in order to address this problem. In general, most of these strategies have fallen into two basic formulation methods that are commonly used for coupling the dynamics of the liquid and the gaseous phase: the Euler-Lagrangian method and the Euler-Eulerian method. The Euler-Lagrangian [16] method has been used by many researchers and various improvements to the basic scheme have been proposed [17-21]. Over recent years the Discrete Droplet Model (DDM) within the Euler-Lagrangian framework has dominated in predicting the behaviour of the spray process. In this method, the spray is represented by finite number of droplet groups, called droplet parcels. It is assumed that all the droplets within one parcel are similar in size and have the same physical properties. The motion and transport of each parcel is tracked through the flow field using the Lagrangian formulation, whilst the gaseous phase is described solving the conservation equations using the Eulerian formulation. The coupling between the liquid and the gaseous phase is taken into account by introducing appropriate source terms for interfacial mass, momentum and energy exchange [22]. Although various researchers and engineers have used the Euler-Lagrangian formulation as a numerical simulation tool for predicting the characteristics of complex multiphase droplet flows to guide their engineering devices designs, the concepts and applications have severe limitations. This formulation is very sensitive to the grid resolution in the near nozzle region [23] and reveals limitations in the descriptions of dense sprays. This assumes that the spray is sufficiently diluted; usually the discrete phase volume fractions should be less than 10\%. It also shows statistical convergence problems, as discussed by [24,25]. Thus, the Euler-Lagrangian formulation is most often used to reliably describe sprays produced by low pressure atomisation [26]. Above mentioned difficulties could be overcome by a stronger physical coupling of the gaseous and liquid phases using the Euler-Eulerian formulation. This method treats the liquid phase and the gaseous phase as interpenetrating continua where both phases are treated from the Eulerian point of view. Hence, this method neglects the discrete nature of the dispersed phase and approximates its effects upon the continuous phase. The same discretisation, and similar numerical techniques and conservation equations are used for both phases. This method was first addressed by [27]. The Euler-Eulerian method has been adopted by a number 
of researches and applied for numerical simulation, e.g. [28-32]. Compared to the Lagrangian scheme, the Eulerian scheme calculation is fairly efficient for flows with high droplet concentration, whilst the Lagrangian scheme generally requires a large number of parcels in each control volume of the calculation domain, particularly for unsteady calculations. However, in order to better capture the behaviour of spray and characteristics of droplets in a dense region using the Eulerian framework, the droplet-size distribution has to be divided into a number of separate size classes ( $\mathrm{n}$ liquid phases). The complete set of conservation equations, with additional source terms accounting for the droplet dynamics, has to be solved for each liquid phase under consideration. This leads to a great number of conservation equations that have to be solved in order to properly describe the spray behaviour. A higher number of liquid phases means better spray resolution, but on the other hand, computational effort is significantly increased.

For modelling the combustion process in IC engine, it is of great importance to reliably describe the fuel-air mixing phenomena. Therefore, the focus in this manuscript is on application and validation of the Eulerian multiphase approach used for modelling the high pressure diesel spray, especially fuel-air mixing and fuel penetration. The numerical simulations of the sprays performed in this work were based on the Eulerian multiphase method which was integrated into the commercial CFD code AVL FIRE ${ }^{\circledR}$. Validation of the Eulerian multiphase spray model against the experimental data was carried out for further improving and developing the physical spray models within the code. Several simulations of high pressure diesel injections combined with different chamber pressures using an Eulerian multiphase method with fixed droplet size classes were carried out. Linking the implemented models with CFD code was performed by using the common FORTRAN 90 routines.

The complete spray model, including the primary and secondary disintegration, droplet collision and evaporation processes is presented. The model showed a capability to predict the strong impact of rail pressure on penetration of the fuel vapour and of the fuel liquid jet.

\section{Eulerian multiphase approach}

As mentioned, the basis of the Eulerian approach is a multiphase method obtained through the ensemble averaging process of the conservation equations [33]. Both the gaseous and the liquid phase are treated as interpenetrating continua characterised by their volume fraction. Each control volume is occupied by phases where each phase takes a certain part of the control volume defining its volume fraction. The first phase is defined as the gaseous phase consisting of gas and fuel vapour mixture. The phases denoting from 2 to $n-1$ are the droplet size classes represented by the droplet diameter whilst the last phase $\mathrm{n}$ is the bulk liquid phase emerging from the nozzle hole.

The set of conservation equations is solved for each phase separately. This leads to a greater number of equations which need to be solved in order to describe the spray behaviour. The degree to which the spray description is accurate depends on the number of liquid phases, where a higher number of droplet phases means better spray resolution but also greater computational effort.

Eqs. (1)-(3) show the mass, momentum, and enthalpy conservation equations for phase $k$ [34]. The terms on the right hand side of the conservation equations $\Gamma_{k l}, \mathbf{M}_{k l}$ and $H_{k l}$, are the mass, momentum and enthalpy exchange terms between phases $k$ and $l$. These terms contain the appropriate physics of the spray model, whilst the left hand side determines the rate of change and the convective transport of the phase flow properties. Eq. (4) represents the volume fraction compatibility condition that must be fulfilled as a prerequisite of the conservative approach [34].

$$
\begin{aligned}
\frac{\partial \alpha_{k} \rho_{k}}{\partial t}+\nabla \cdot\left(\alpha_{k} \rho_{k} \mathbf{v}_{k}\right)=\sum_{l=1, l \neq k}^{n} & \Gamma_{k l} \\
\frac{\partial \alpha_{k} \rho_{k} \mathbf{v}_{k}}{\partial t}+\nabla \cdot\left(\alpha_{k} \rho_{k} \mathbf{v}_{k} \mathbf{v}_{k}\right)= & -\alpha_{k} \nabla p+\nabla \cdot \alpha_{k}\left(\tau_{k}+\tau_{k}^{t}\right)+\alpha_{k} \rho_{k} \mathbf{f} \\
& +\sum_{l=1, l \neq k}^{n} \mathbf{M}_{k l}+\mathbf{v}_{k} \sum_{l=1, l \neq k}^{n} \Gamma_{k l} \\
\frac{\partial \alpha_{k} \rho_{k} h_{k}}{\partial t}+\nabla \cdot\left(\alpha_{k} \rho_{k} \mathbf{v}_{k} h_{k}\right)= & \nabla \cdot \alpha_{k}\left(\mathbf{q}_{k}+\mathbf{q}_{k}^{t}\right)+\alpha_{k} \rho_{k} \mathbf{f} \cdot \mathbf{v}_{k} \\
& +\alpha_{k} \rho_{k} \theta_{k}+\alpha_{k} \tau_{k}: \nabla \mathbf{v}_{k}+\alpha_{k} \frac{d p}{d t} \\
& +\sum_{l=1, l \neq k}^{n} \mathrm{H}_{k l}+h_{k} \sum_{l=1, l \neq k}^{n} \Gamma_{k l}
\end{aligned}
$$

$\sum_{k=1}^{n} \alpha_{k}=1$

For description of highly turbulent spray behaviour, the $k-z e t a-f$ turbulence model was employed [35]. This model is based on Durbin's elliptic relaxation concept and it solves the transport equation for the velocity scale ratio $\zeta=v^{2} / k$, making the model robust and suitable for spray calculations. The turbulence model equations are shown in Eqs. (5)-(8).

$$
\begin{aligned}
& \rho \frac{D k}{D t}=\rho\left(P_{k}-\varepsilon\right)+\frac{\partial}{\partial x_{j}}\left[\left(\mu+\frac{\mu_{t}}{\sigma_{k}}\right) \frac{\partial k}{\partial x_{j}}\right] \\
& \rho \frac{D \varepsilon}{D t}=\rho \frac{C_{\varepsilon 1}^{*} P_{k}-C_{\varepsilon 2}^{*} \varepsilon}{T}+\frac{\partial}{\partial x_{j}}\left[\left(\mu+\frac{\mu_{t}}{\sigma_{\varepsilon}}\right) \frac{\partial \varepsilon}{\partial x_{j}}\right] \\
& \rho \frac{D \varsigma}{D t}=\rho f-\rho \frac{\varsigma}{k} P_{k}+\frac{\partial}{\partial x_{j}}\left[\left(\mu+\frac{\mu_{t}}{\sigma_{\varsigma}}\right) \frac{\partial \varsigma}{\partial x_{j}}\right]
\end{aligned}
$$

$f-L^{2} \frac{\partial^{2} f}{\partial x_{j} \partial x_{j}}=\left(C_{1}+C_{2} \frac{P_{k}}{\varsigma}\right) \frac{(2 / 3-\varsigma)}{T}$

The liquid jet penetration is mainly influenced by the thermodynamic conditions of the gas into which the spray penetrates and therefore the influence of the cavitation was not investigated. The interfacial mass exchange term $\Gamma_{k l}$ gets its contributions from primary break-up $P$, secondary break-up $S$, droplet collisions $C$ and droplet evaporation $E$, as shown in Eq. (9).

$\Gamma_{k l}=\Gamma_{P, k l}+\Gamma_{S, k l}+\Gamma_{C, k l}+\Gamma_{E, k l}$

The mass exchange between two phases acts in both directions, so that one can write $\Gamma_{\text {Process }, k l}=-\Gamma_{\text {Process, } l k}$. This means that a mass gain for phase $k$ is a mass loss for phase $l$ and vice versa, ensuring the overall mass conservation. In order to predict the fuel mass fraction, $Y_{i}$, an additional transport equation is solved for the gaseous phase, as can be seen in Eq. (10).

$$
\frac{\partial}{\partial t} \alpha_{1} \rho_{1} Y_{i}+\nabla \cdot\left(\alpha_{1} \rho_{1} \mathbf{v}_{1} Y_{i}\right)=\nabla \cdot\left(\alpha_{1}\left(\rho_{1} \mathrm{D}_{Y_{i}}+\frac{\mu_{1}^{\mathrm{t}}}{\mathrm{Sc}^{t}}\right) \nabla Y_{i}\right)+S_{Y_{i}} .
$$

The source term $S_{Y_{i}}$ is determined by collecting the evaporated liquid mass from all droplet phases denoting from 2 to $n$, implying that the evaporation takes place between all liquid phases and the 
gaseous phase. The interfacial mass exchange rate due to the evaporation between the droplet phase $k$ and the gaseous phase 1 is determined by Eq. (11):

$\Gamma_{E, k 1}=-N_{k} \dot{m}_{E, k 1}=-\Gamma_{E, 1 k}$,

where the droplet number density $N_{k}$ denotes the number of droplets per unit volume. Assuming spherical droplets with diameter $D_{k}, N_{k}$ can be derived from the Eq. (12):

$\frac{\pi D_{k}^{3}}{6} N_{k}=\alpha_{k} \rightarrow N_{k}=\frac{6 \alpha_{k}}{\pi D_{k}^{3}}$.

The term $\dot{m}_{E, k 1}$ in Eq. (11) stands for the evaporated mass exchange rate of a single droplet calculated according to the model of [36]. The negative sign results from the sign convention of the evaporation model where a mass loss due to the evaporation has a positive sign.

Fig. 1 shows the scheme of the break-up behaviour contributing to the terms $\Gamma_{P, k l}$ and $\Gamma_{S, k l}$ presented in the Eq. (9). The droplet classes are sorted in the ascending manner, where phase 2 denotes the droplet phase with the smallest size class diameter. The bulk liquid phase $n$, defined with a diameter equal to the nozzle hole diameter, firstly disintegrates into the droplet phases denoting from 2 to $n-1$ due to the primary break-up process. The created droplets are then subject to further secondary disintegration into droplets with even smaller diameters due to the aerodynamic and turbulent forces.

Fig. 2 shows a blob injection model with a decreasing number of blobs, which is used to model the primary break-up process. The mass loss of the parent droplet results in a reduction of the phase volume fraction $\alpha_{n}$ leading to reduction in the number of blobs $N_{n}$, leaving the size class diameter $D_{n}$ constant.

The mass exchange rate due to the primary and secondary break-up, $\Gamma_{P, n l}$ and $\Gamma_{S, k l}$, are determined by the diameter change rates $\left(d D_{n} / \Delta t\right)_{P, n l}$ and $\left(d D_{k} / \Delta t\right)_{S, k l}$, resulting from the used models. Eq. (13) denotes the general break-up mass exchange rate, where

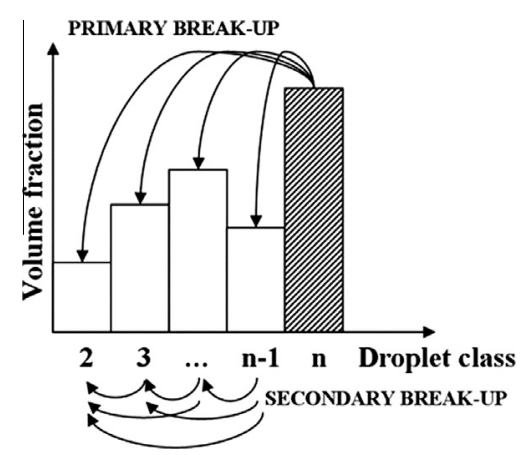

Fig. 1. Mass exchange due to primary and secondary break-up processes.

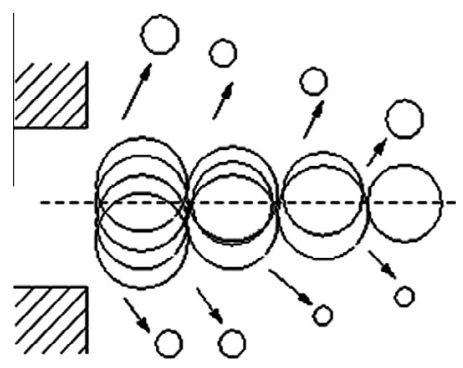

Fig. 2. Blob injection with decreasing number of blobs. subscript $B r$ is replaced with $P$ for primary and with $S$ for calculating the secondary break-up process.

$\Gamma_{B r, n l}=\frac{1}{2} N_{n} \rho_{n} \pi D_{n}^{2}\left(\frac{d D_{n}}{d t}\right)_{B r, n l}=\frac{3 \alpha_{n} \rho_{n}}{D_{n}}\left(\frac{d D_{n}}{d t}\right)_{B r, n l}=-\Gamma_{B r, l n}$.

The disintegration rate of the bulk liquid phase $n$ into the droplet phase $l,\left(d D_{n} / \Delta t\right)_{P, n l}$, is calculated according to the model of [37], taking into consideration two independent mechanisms aerodynamic surface wave growth and internal turbulence stresses caused by injector flow. The diameter change rate of the blob due to the secondary break-up $\left(d D_{k} / \Delta t\right)_{S, k l}$ is modelled by the standard WAVE model [38]. These models are applied in each cell of the spray region where the liquid volume fraction exceeds the defined threshold value. If the predicted size of the break-up products $D_{l}$ is less than the parent droplet diameter $D_{k}$, mass is transferred according to the break-up rate into the corresponding droplet size class. The model parameterisation was performed by analysing the influence of the model coefficients $B_{S 1}$ and $B_{S 2}$ for the secondary atomisation model and coefficients $B_{P 1}, B_{P 2}$ and $B_{P 3}$ for the primary atomisation model [39]. The coefficients used within the atomisation models are presented by Eqs. (14)-(17), whilst the break-up model details are described in [40]. The definition of the harmonic turbulent/aerodynamic time scale combination is shown in [34].

$\tau_{A}=\left[\frac{1}{B_{P 1} \cdot \tau_{T}}+\frac{1}{B_{P 3} \cdot \tau_{W}}\right]^{-1}$

$L_{T}=B_{P 2} C_{\mu} \frac{k_{\text {avg }}^{1.5}}{\varepsilon_{\text {avg }}}=L_{A}$

$R_{t}=\Lambda_{n} B_{S 1}$

$\tau_{a}=\frac{3.726 B_{S 2} R_{n}}{\Lambda_{n} \Omega_{n}}$

The rate of the primary atomisation is defined using the overall atomisation time scale $\tau_{A}$, which is furthermore defined as a harmonic combination of the turbulent and aerodynamic time scales, $\tau_{T}$ and $\tau_{W}$. This influence is visible in Eq. (14). The diameter of the droplet generated due to the primary atomisation depends on the atomisation length scale $L_{A}$, as seen in Eq. (15). After the primary atomisation, the droplets with the target diameter $R_{t}$ are created due to the secondary atomisation. The target diameter is a function of the instability wavelength and the coefficient $B_{S 1}$, as shown with Eq. (16). The final expression for the secondary atomisation rate is defined according to Eq. (17), where $R_{n}, \Lambda_{n}$ and $\Omega_{n}$ are the diameter, wavelength and growth rate for the observed droplet phase $n$.

The mass exchange due to the droplet collision process is modelled according to the stochastic collision model derived by O'Rourke [41]. This model uses relative droplet velocity to calculate interfacial collision frequency. It was adopted for the Eulerian framework and implemented within the commercial CFD code [42]. The collision mass sources are modelled according to Eq. (18):

$\Gamma_{C, i}=\Gamma_{C, k}+\Gamma_{C, l}$,

where the term on the left side is the mass source of the created droplet. The terms on the right hand side stand for the mass sources of the bigger and smaller droplets, respectively. The final expression for mass sources is given in Eqs. (19) and (20).

$$
\begin{aligned}
& \Gamma_{C, k}=\frac{d_{k}^{3} \pi}{6} \rho N_{k} \frac{1}{\Delta t} \\
& \Gamma_{C, l}=\frac{d_{l}^{3} \pi}{6} \rho N_{k} N_{\text {collis }}
\end{aligned}
$$


Terms $d_{k}$ and $d_{l}$ are the diameters of the colliding droplet phases, the term $\rho$ stands for the liquid fuel density, term $N_{k}$ is the number of droplets in a certain control volume for phase $k$ and the term $\Delta t$ is the calculation time step. The last term in the Eq. (20) is the modelled number of interfacial droplet collisions. The phase indexes $k$ and $l$ cannot be equal to each other and cannot have values 1 or $n$. This arises from the constraint that collision is not considered for the bulk liquid, nor for the gaseous phase. The mass sources of the colliding phases are transferred to a target droplet phase $i$ and mass, momentum and enthalpy are conserved. Currently, the droplet coalescence is modelled as the collision only outcome. One drawback of this model is the impossibility of predicting the collision between droplets contained within one droplet size class due to the lack of relative velocity. The interfacial momentum exchange between the gaseous and the liquid phases $\mathbf{M}_{k 1}$ is determined by drag $\mathbf{M}_{D, k 1}$ and turbulent dispersion forces $\mathbf{M}_{T, k 1}$, as shown with Eq. (21).

$$
\begin{aligned}
\mathbf{M}_{k 1} & =\mathbf{M}_{D, k 1}+\mathbf{M}_{T, k 1} \\
& =c_{D} \frac{6 \alpha_{k} \rho_{1}}{D_{k}}\left|\mathbf{v}_{1}-\mathbf{v}_{k}\right|\left(\mathbf{v}_{1}-\mathbf{v}_{k}\right)+c_{T} \rho_{1} k_{1} \nabla \alpha_{k}=-\mathbf{M}_{1 k}
\end{aligned}
$$

The drag coefficient $c_{D}$ is a function of the droplet Reynolds number and the liquid volume fraction. The turbulent dispersion force is modelled following the method of [43], with a constant or modelled turbulent dispersion force coefficient $c_{T}$.

Table 1

The phase specification of the Eulerian spray approach.

\begin{tabular}{lcccccc}
\hline Case & $a$ & $b$ & $c$ & \multicolumn{1}{c}{$d$} & \multicolumn{1}{c}{$e$} & \multicolumn{1}{c}{$f$} \\
\hline Injection pressure (MPa) & 50 & 50 & 80 & 80 & 120 & 120 \\
Chamber pressure (MPa) & 5.4 & 7.2 & 5.4 & 7.2 & 5.4 & 7.2 \\
\hline
\end{tabular}

The evaporation model determines the interfacial enthalpy exchange $\mathrm{H}_{k 1}$ between the gaseous phase and the liquid phases. In Eq. (22) the term $\dot{Q}_{E, k 1}$ represents the heat flow rate into a single droplet and is calculated according to the correlation of [36].

$\mathrm{H}_{k 1}=N_{k} \dot{Q}_{E, k 1}=\frac{6 \alpha_{k}}{\pi D_{k}^{3}} \dot{Q}_{E, k 1}=-\mathrm{H}_{1 k}$.

\section{Simulation setup and experimental data}

Numerical simulations of fuel injection were performed by using the Euler-Eulerian multiphase approach. Six different operating points of high pressure diesel sprays were investigated, as can be seen in Table 1 . In order to achieve mesh independency several computational meshes were examined. Fig. 3 shows the domain used in this research together with the boundary surfaces. For the mesh dependency test, three meshes were generated consisting of 1400,2880 and 5860 control volumes. After evaluating the flow field and droplet distribution, a domain of 1400 control volumes was selected for further research. Mesh was generated as a two-dimensional static computational mesh, extending from 0 to $120 \mathrm{~mm}$ in axial direction and from 0 to $25 \mathrm{~mm}$ in radial direction. Such mesh is used to reduce the CPU time necessary to perform high number of CFD simulations necessary for the model parameterisation. Using the two-dimensional computational mesh is reasonable when the symmetric spray is assumed. The symmetry boundary condition (1) was applied in the tangential direction and the mesh was refined towards the spray inlet (3) and spray axis (4). At the outlet of the domain, the pressure boundary condition was applied (2). The injector surface was defined with the constant temperature $(900 \mathrm{~K})$ wall boundary condition.

Nine Eulerian phases were defined for the spray simulations one gaseous phase, seven droplet phases and one bulk liquid phase.
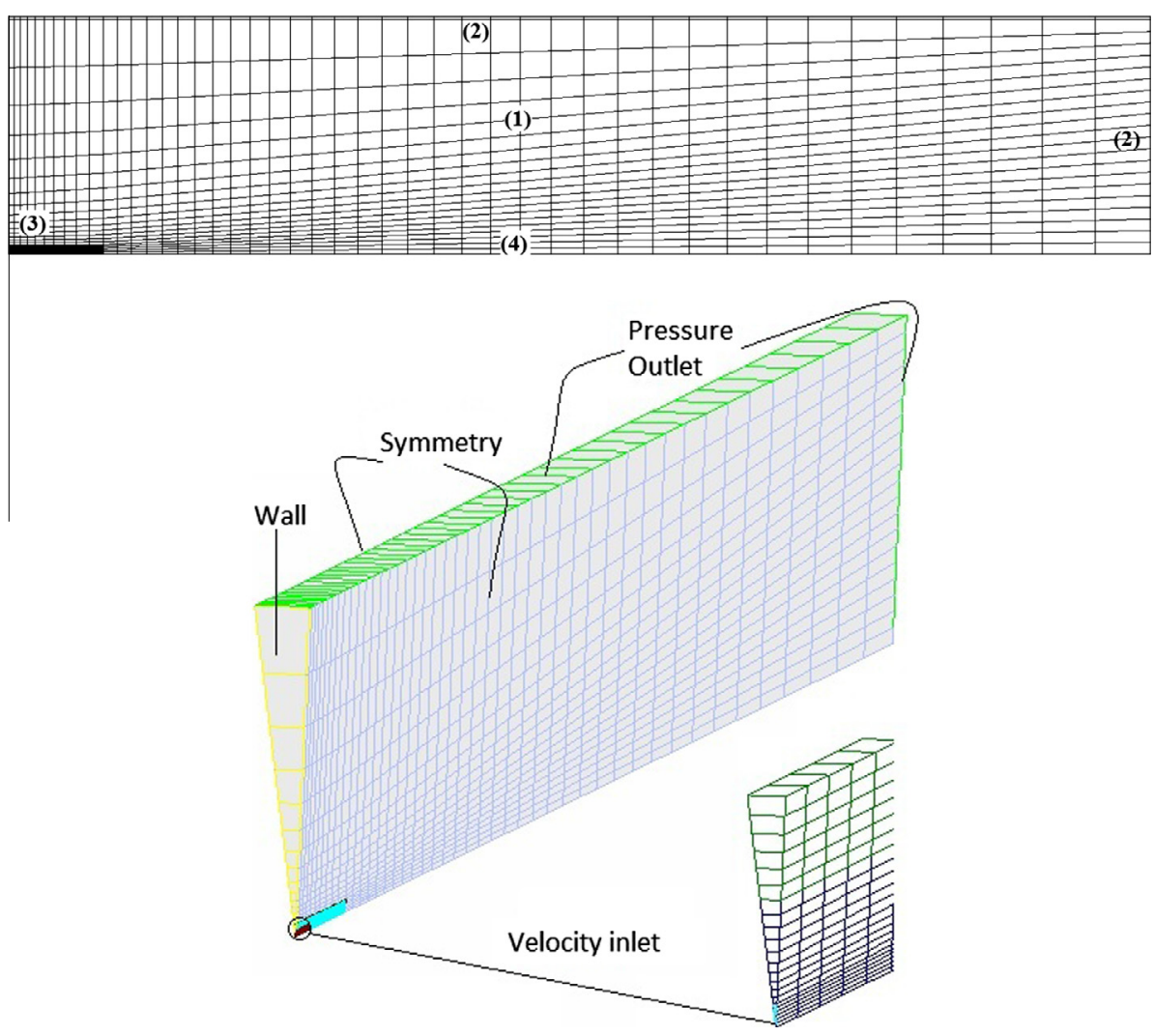

Fig. 3. Computational mesh for numerical investigation. 

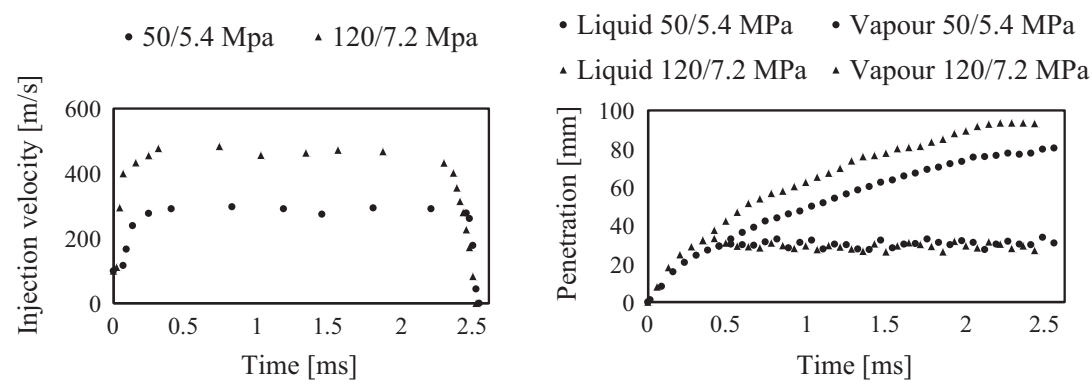

Fig. 4. Experimental results on injection velocity (left) and fuel penetration (right).
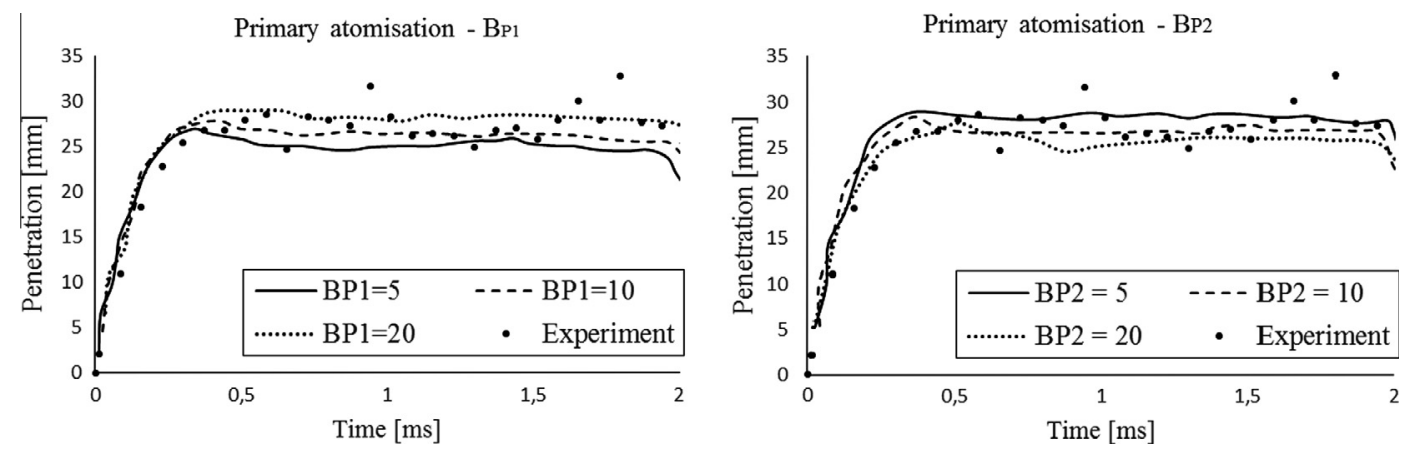

Fig. 5. Primary atomisation parameterisation, case d.

The corresponding diameters were defined with sizes of 5, 10, 20, $40,70,95,140 \mu \mathrm{m}$ (droplet phases) and a diameter of $205 \mu \mathrm{m}$ was assigned to the bulk liquid phase. The experimental investigations were performed at DaimlerChrysler Research within the framework of the European funded I-LEVEL project. The diesel fuel was injected into the high pressure chamber through the 1-hole nozzle with an orifice diameter of $205 \mu \mathrm{m}$ into an $\mathrm{N}_{2}$ environment. The flow within the nozzle is controlled through the fast opening and closing of the needle valve and shaped by the nozzle itself. Fig. 4 shows the experimental data for the selected cases. It is visible that the higher pressure injection results in a more intensive penetration of the vapour phase, whilst the liquid fuel penetration stays at a similar level. On the other hand, the fuel jet velocity is dependent on the injection pressure, which is visible on the left hand side of the Fig. 4. The maximum fuel injection velocities are in the range from 300 to $500 \mathrm{~m} / \mathrm{s}$, depending on the injection pressure. The inlet velocities were imposed according to the experimental data from measured injection rates, which were normalised in order to eliminate the measurement fluctuations. The diesel fuel with temperature of $373 \mathrm{~K}$, density $755 \mathrm{~kg} / \mathrm{m}^{3}$, specific heat $2394 \mathrm{~J} / \mathrm{kgK}$ and molar viscosity equal to $5.123 \mathrm{e}-04 \mathrm{~kg} / \mathrm{ms}$ was used as diesel fuel in this research.

For the turbulence, volume fraction and energy transport equations a first order UPWIND differencing scheme was applied, whilst for the continuity equation the central differencing scheme (CDS) was employed. The CDS can generate numerical oscillations yielding unbounded and non-monotonic solutions. Therefore, for the momentum equation a combination of CDS and UPWIND was proposed by introducing the blending factor of 0.5 [34]. For all calculations the implicit time integration was employed ensuring unconditional solution stability whilst the accuracy was achieved by employing sufficiently small time step. The influence of the false diffusion on the penetration results is minimised by performing mesh dependency analysis. The turbulence was modelled using the advanced $k$-zeta $-f$ turbulence model. Of particular advantage is that it is sufficiently robust to be used for computations involving grids with moving boundaries and highly compressed flows as it is the case in internal combustion engines. It guarantees the good solution for any computational mesh regarding robustness, computing time and accuracy. The solution convergence criterion is achieved when the momentum, pressure, energy and volume fraction residuals decrease under the value of $1 \mathrm{e}-4$. The pressure velocity coupling of the momentum and continuity equation was obtained using the SIMPLE algorithm. The time discretisation used for simulation varied with simulation time and small time-steps were used due to the fact that gradients in the mass exchange models can be very high. Therefore, at the beginning of injection the time-step was very small (3e-08 s) but it was continuously increased throughout the injection time (up to 5e-07 s).

\section{Results and discussion}

Case $d$ with the injection conditions described in Table 1 was used as the reference case for model parameterisation. The primary breakup model coefficient $B_{P 1}$ influences the turbulent time scale, $B_{P 2}$ dictates the turbulent length scale, whilst the $B_{P 3}$ defines the aerodynamic length scale influence on the penetration. The overall atomisation time scale is defined as the harmonic combination of the turbulent and aerodynamic time scale, allowing bigger influence of the faster disintegration process. Higher values of coefficient $B_{P 1}$ result in a slower jet disintegration process causing more intensive tip penetration, as can be seen in Fig. 5. This can be addressed to the prevalence of bigger droplets possessing higher momentum. The same conclusion may be drawn for the influence of $B_{P 3}$ coefficient and therefore it was not shown. The change in coefficient $B_{P 2}$, which influences the mass exchange rate, has the opposite effect on the spray temporal distribution. Increasing the exchange rate the jet penetration decreases due to the higher diameter change rate and the creation of smaller droplets which are being more influenced by the drag force. 

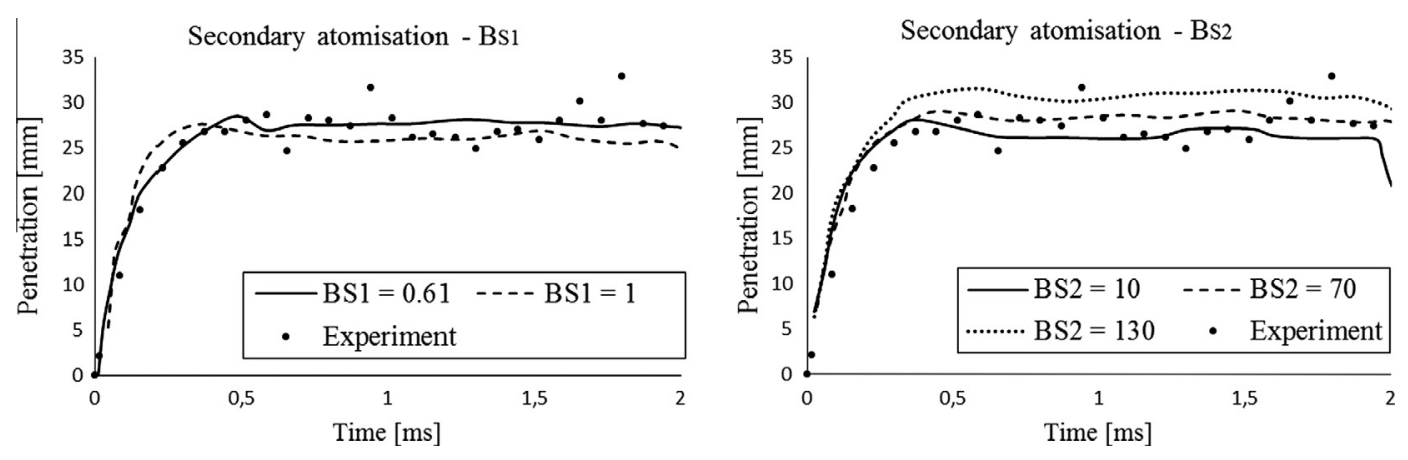

Fig. 6. Secondary atomisation parameterisation, case d.
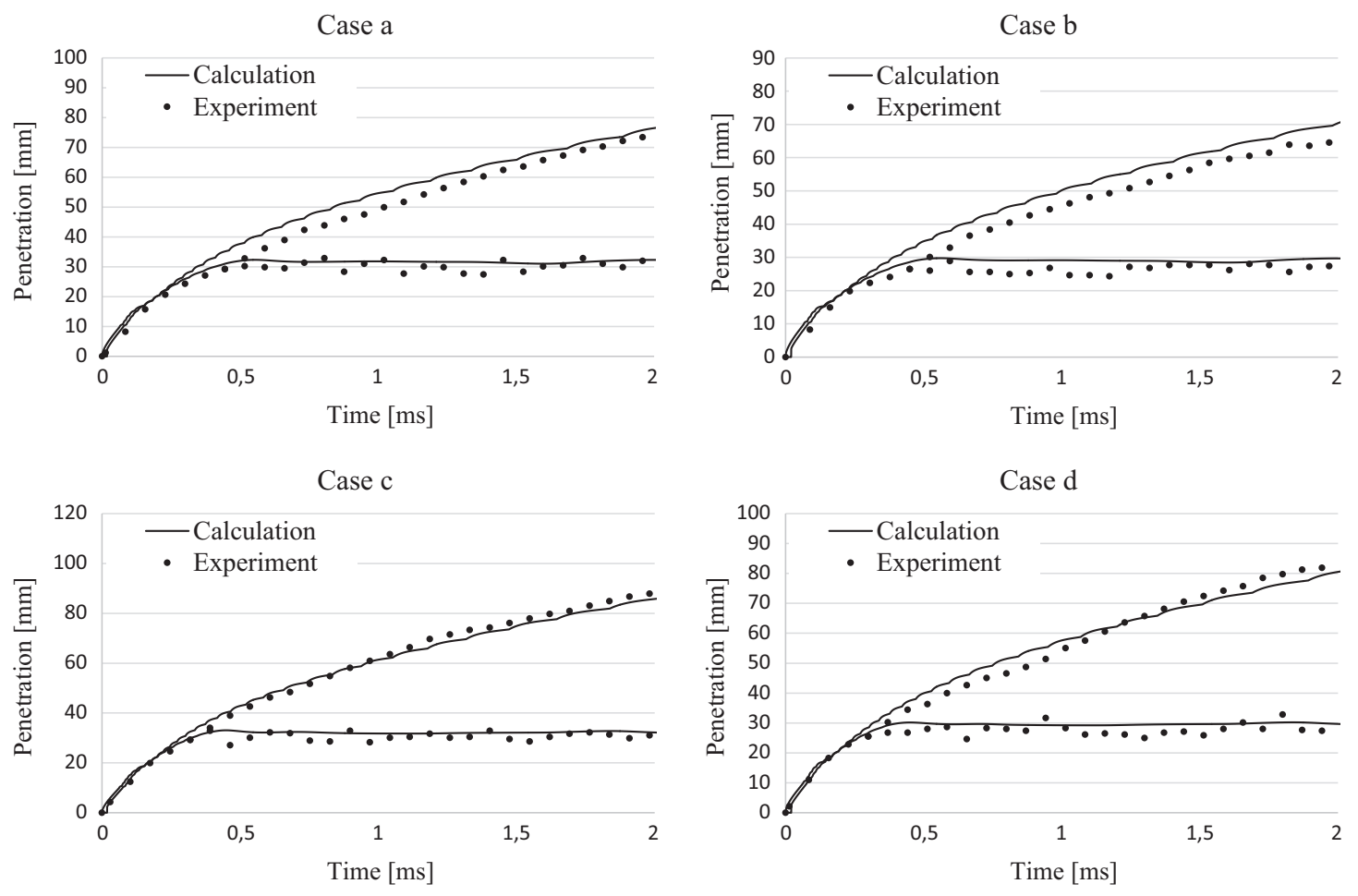

Case e
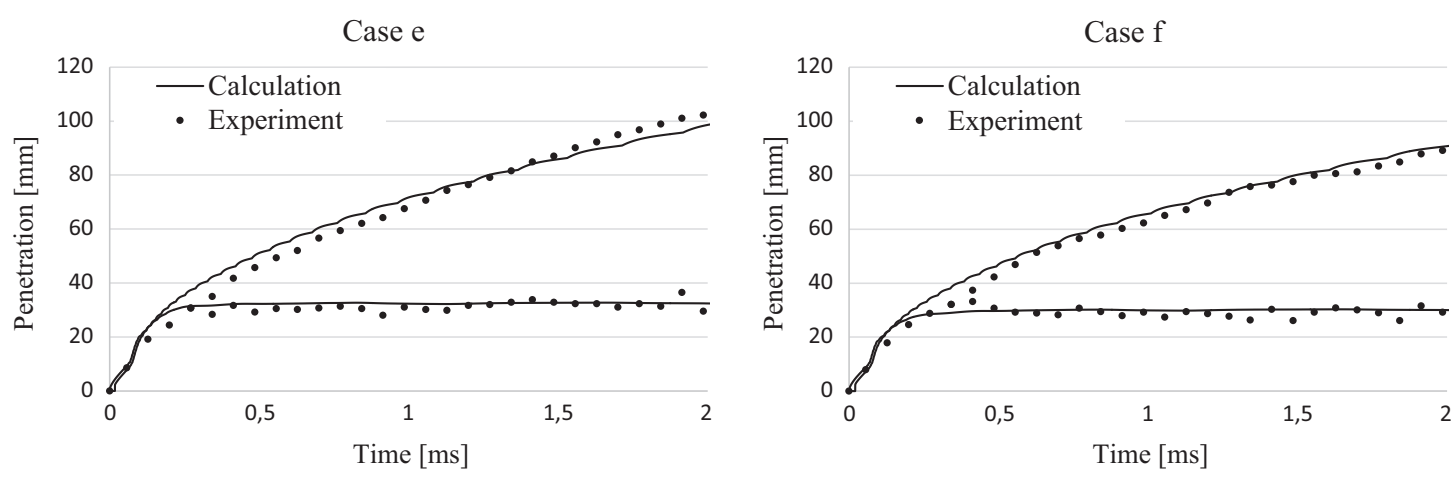

Fig. 7. Liquid fuel and vapour penetration for conditions a-f.

The model for secondary atomisation takes into account the occurrences of surface instabilities caused by the aerodynamic forces. The diameter of the created droplet due to such effect is defined with the wavelength of the fastest growing surface wave. Correlation between the diameter and the wavelength is defined by the atomisation coefficient $B_{S 1}$. Higher values result in bigger droplets. The restriction of this coefficient within the boundaries
0.61 and 1 is recommended. To take into account nozzle influence on the secondary atomisation another coefficient, $B_{S 2}$, is introduced. With increasing the value of $B_{S 2}$ the atomisation time is prolonged and the tip reaches further into the domain. The influence of the secondary atomisation model coefficients is shown in Fig. 6.

Fig. 7 shows the comparisons between the calculated and measured liquid and vapour spray tip penetration curves for all of the 

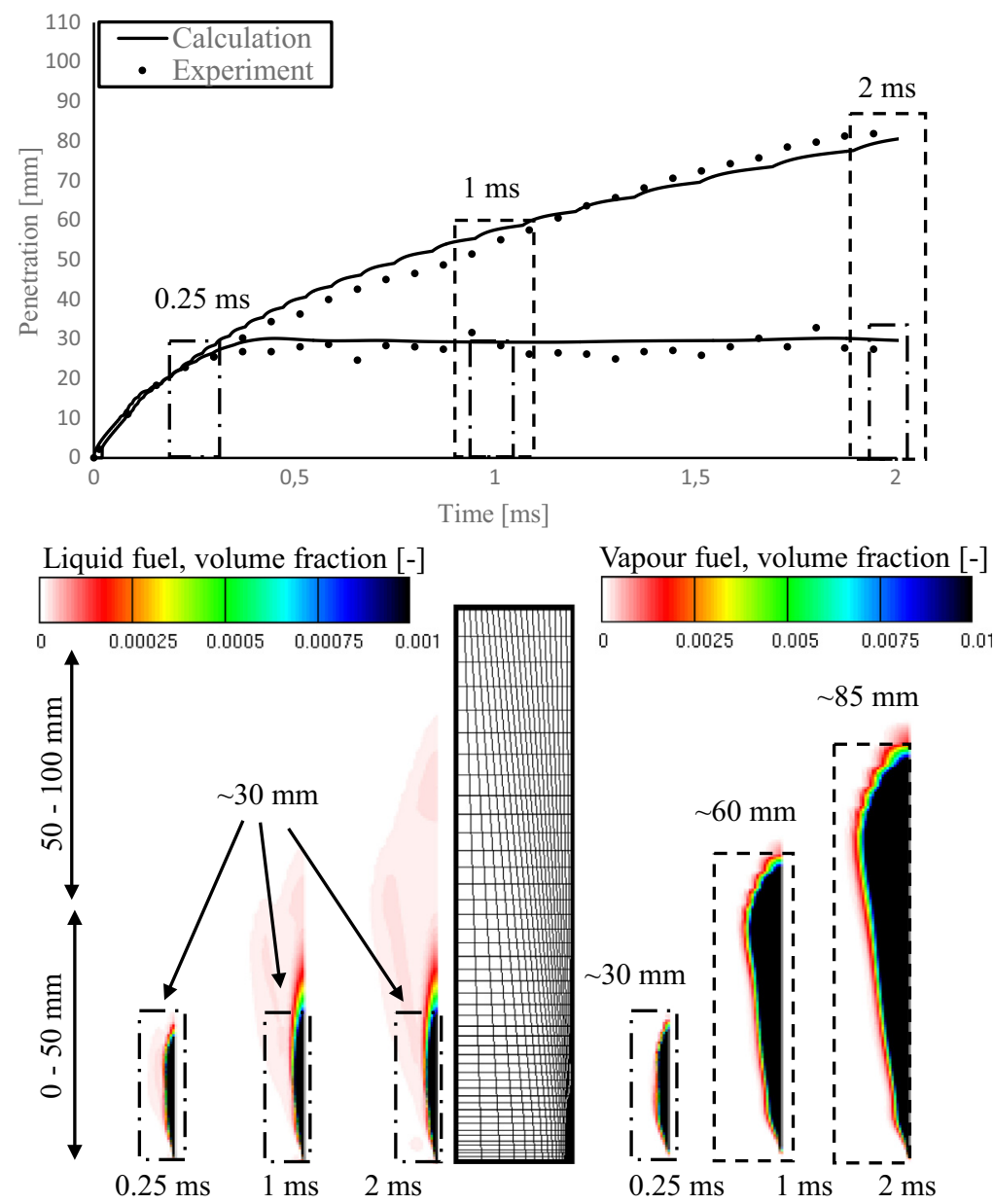

Fig. 8. CFD results of liquid fuel and vapour penetration for case d.

examined cases. The method with fixed droplet size classes was employed in these simulations. The black solid line represents the simulation results with the activated collision, primary atomisation, secondary atomisation, and evaporation models. The results of the experimental research are represented with the black dots and black triangles. The injection was predefined with the inlet boundary conditions. As shown in Fig. 4, the hat-shape velocity profile was assumed normal to the nozzle hole. The fuel started to penetrate rapidly, reaching high velocity, and the primary atomisation process occured. This approximation was valid for the jet penetration research but for investigating other spray characteristics and the combustion process a more detailed inlet boundary description should be obtained. The flow differences arose from the needle movement and the nozzle hole geometrical imperfections, so a proper approach would be to measure the needle lift movement, including axial/radial displacement and calculate fuel flow through the nozzle to generate the real conditions of the nozzle hole, which take into account the turbulence and cavitation processes. The liquid fuel started to evaporate and transferred a certain momentum to the vapour phase, ensuring the same penetration velocity for both phases. At this point it is important to mention that the detection of the spray contour in the Euler-Eulerian spray simulation was more difficult than for the Lagrangian spray model. Here, the spray contours were determined by the limiting threshold values for the liquid volume fraction and the vapour mass fraction. More details about the determination process of the limiting threshold values can be found in [40]. In our work, the liquid tip penetration was defined as the furthest distance of the liquid phase where the total liquid volume fraction accounts for $0.1 \%$ of the control volume. With regard to the vapour penetration, a threshold value of $1 \%$ was defined. At the certain time after the injection, approximately at $0.4 \mathrm{~ms}$, the liquid droplets located at the jet tip completely evaporated and the liquid volume fraction dropped below the threshold value. Complete evaporation for the defined injection parameters was achieved at approximately $30 \mathrm{~mm}$ from the nozzle hole in the spray axis direction. The given results imply that the vapour penetration was reduced with increase in the ambient density due to the higher momentum dissipation. Furthermore, the vapour penetrated further into the domain due to increase in injection pressure and jet velocity.

Fig. 8 shows the results of diesel injection at $50 \mathrm{MPa}$ rail pressure and 5.4 MPa chamber pressure. It is visible that the predicted penetration is in a good agreement with the measurements, indicating that the number of liquid phases and sub-models accounting for the appropriate physics of spray were chosen correctly. On the upper side of Fig. 8 the calculated liquid and vapour penetration compared to the experimental data is shown. As can be seen, the developed spray had a liquid penetration at around $30 \mathrm{~mm}$ along the spray axis. Liquid (left) and vapour (right) volume fraction simulation results are shown at the bottom of the Figure. At the injection time of $0.25 \mathrm{~ms}$, the spray was still in the transitional period. The evaporated fuel followed the liquid core penetration and the penetration curves overlapped. Reaching the developed spray state where all the liquid fuel evaporated, the liquid penetration stagnated and oscillated around the developed liquid penetration length. As is visible at $1 \mathrm{~ms}$ and $2 \mathrm{~ms}$ after the start of injection, the fuel vapour continued to penetrate due to 
the momentum transferred from the injected liquid fuel. In addition, a shift of the vapour mass from the spray axis in the radial direction is visible due to the turbulent dissipation. In Fig. 8 the colour bar is set to have maximum values of $1 \%$ and $0.1 \%$ of the volume fraction for the vapour and the liquid phase, respectively. Therefore, the black area represents the control volumes at which the volume fractions of the desired phase exceeded the threshold values corresponding to the previously defined penetration length.

\section{Conclusion}

The overall engine performance in terms of fuel consumption and emissions is highly influenced by the spray processes, and thus an understanding of these processes is essential in engine development. An Eulerian multiphase spray modelling concept, applying the method with constant size class diameters assigned to each of the liquid droplet phases, has been presented. The primary breakup, secondary breakup, collision and evaporation models were utilised to adequately describe six cases with different injection and chamber pressure combinations. The influence of pressure variation on spray development was clearly visible. The increase in the injection pressure caused further fuel vapour penetration with only minor influences on the fuel liquid penetration, whilst the increase in chamber pressure had the opposite effect. Due to the higher surrounding gas mixture pressure and increased density, a larger spray angle with lower tip penetration was observed. The set of model coefficients was derived by performing the parameterisation on the reference case, where the individual influence of the coefficients on the spray propagation was examined. Change in the model coefficients influenced the droplet size and the atomisation time, and as a result different temporal and spatial droplet-vapour distributions were achieved. The validation of the Eulerian approach was conducted by comparing the calculation results against available experimental data. Overall, it can be said that the Eulerian multiphase spray model adequately described the liquid and fuel vapour penetration in comparison with all six experimental cases, covering a wide range of high pressure injection conditions. The Eulerian multiphase spray model showed the capability of predicting the strong impact of rail pressure on the penetration of the fuel vapour and liquid fuel. Furthermore, this research showed that using the Eulerian multiphase is a good approach for adequately describing highly turbulent diesel fuel injection processes and can serve as valuable tool in the process of development of modern fuel injection systems.

\section{Acknowledgements}

The authors wish to thank the AVL List GmbH, Graz, Austria for the financing and opportunity to work on the research project. The authors also wish to thank the CFD development group at AVL-AST, Graz, Austria, for their continuous support and useful technical discussions during the development of numerical models. The experimental spray data was measured by Dr. Gerhard König at the DaimlerChrysler Research Department.

\section{References}

[1] Klemeš II, Varbanov PS, Huisingh D. Recent cleaner production advances in process monitoring and optimisation. J Clean Prod 2012;34:1-8. http:/1 dx.doi.org/10.1016/i.jclepro.2012.04.026.

[2] Niemisto I, Saavalainen P, Pongracz E, Keiski RL. Biobutanol as a potential sustainable biofuel - assessment of lignocellulosic and waste-based feedstocks. J Sustain Dev Energy Water Environ Syst 2013;1(2):58-77.

[3] Kozarac D, Vuilleumier D, Saxena S, Dibble RW. Analysis of benefits of using internal exhaust gas recirculation in biogas-fueled $\mathrm{HCCl}$ engines. Energy Convers Manage 2014;87:1186-94. $\underline{\underline{\text { http://dx.doi.org/10.1016/ }}}$ i.enconman.2014.04.085.
[4] Taseska V, Markovska N, Causevski A, Bosevski T, Pop-Jordanov J. Greenhouse gases (GHG) emissions reduction in a power system predominantly based on lignite. Energy 2011;36:2266-70. http://dx.doi.org/10.1016/i.energy.2010.04. $\underline{010 .}$

[5] Mathiesen BV, Lund H, Karlsson K. 100\% Renewable energy systems, climate mitigation and economic growth. Appl Energy 2011;88:488-501. http:/ dx.doi.org/10.1016/i.apenergy.2010.03.001.

[6] Katrašnik T. Hybridization of powertrain and downsizing of IC engine - a way to reduce fuel consumption and pollutant emissions - Part 1. Energy Convers Manage 2007;48:1411-23. http://dx.doi.org/10.1016/i.enconman.2006. 12.004 .

[7] Su L, Li X, Zhang Z, Liu F. Numerical analysis on the combustion and emission characteristics of forced swirl combustion system for DI diesel engines. Energy Convers Manage 2014;86:20-7. http://dx.doi.org/10.1016/i.enconman. 2014.05.023.

[8] Wei S, Wang F, Leng X, Liu X, Ji K. Numerical analysis on the effect of swirl ratios on swirl chamber combustion system of DI diesel engines. Energy Convers Manage 2013;75:184-90. http://dx.doi.org/10.1016/j.enconman. 2013.05 .044 .

[9] Reitz R. Development and testing of diesel engine CFD models. Prog Energy Combust Sci 1995;21:173-96. $\quad$ http://dx.doi.org/10.1016/03601285(95)00003-Z

[10] Choi CY, Reitz RD. An experimental study on the effects of oxvgenated fuel blends and multiple injection strategies on DI diesel engine emissions. Fuel 1999;78:1303-17. http://dx.doi.org/10.1016/S0016-2361(99)00058-7.

[11] Fuster D, Agbaglah G, Josserand C, Popinet S and Zaleski S Numerical simulation of droplets, bubbles and waves: state of the art Fluid Dynamics Research 41;065001.

[12] Sun Z-Y, Li G-X, Chen C, Yu Y-S, Gao G-X. Numerical investigation on effects of nozzle's geometric parameters on the flow and the cavitation characteristics within injector's nozzle for a high-pressure common-rail DI diesel engine. Energy Convers Manage 2015;89:843-61. http://dx.doi.org/10.1016/ i.enconman.2014.10.047.

[13] Kong S-C, Reitz RD. Application of detailed chemistry and CFD for predicting direct injection $\mathrm{HCCl}$ engine combustion and emissions. Proc Combust Inst 2002;29:663-9. http://dx.doi.org/10.1016/S1540-7489(02)80085-2.

[14] Saario A, Rebola A, Coelho P, Costa M, Oksanen A. Heavy fuel oil combustion in a cylindrical laboratory furnace. measurements and modeling. Fuel 2005;84:359-69. http://dx.doi.org/10.1016/i.fuel.2004.10.002.

[15] Bi X, Liu H, Huo M, Shen C, Qiao X, Lee CF. Experimental and numerical study on soot formation and oxidation by using diesel fuel in constant volume chamber with various ambient oxygen concentrations. Energy Convers Manage 2014;84:152-63. http://dx.doi.org/10.1016/j.enconman.2014.04.023.

[16] Mikulčić H, Berg E, Vujanović M, Priesching P, Tatschl R, Duić N. Numerical analysis of cement calciner fuel efficiency and pollutant emissions. Clean Technol Environ Policy 2013;15:489-99. http://dx.doi.org/10.1007/s10098013-0607-5.

[17] Dukowicz JK. A particle-fluid numerical model for liquid sprays. J Comput Phys 1980;35:229-53. http://dx.doi.org/10.1016/0021-9991(80)90087-X.

[18] Gouesbet G, Berlemont A. Eulerian and Lagrangian approaches for predicting the behaviour of discrete particles in turbulent flows. Prog Energy Combust Sci 1999;25:133-59. http://dx.doi.org/10.1016/S0360-1285(98)00018-5.

[19] Loth E. Numerical approaches for motion of dispersed particles, droplets and bubbles. Prog Energy Combust Sci 2000;26:161-223. http://dx.doi.org/ 10.1016/S0360-1285(99)00013-1.

[20] Chen X-Q, Pereira JCF. Stochastic-probabilistic efficiency enhanced dispersion modeling of turbulent polydispersed sprays. J Propuls Power 1996;12:760-9. http://dx.doi.org/10.2514/3.24099.

[21] Hildenbrand S, Staudacher S, Brüggemann D, Beyrau F, Weikl MC, Seeger T, et al. Numerical and experimental study of the vaporization cooling in gasoline direct injection sprays. Proc Combust Inst 2007;31:3067-73. http://dx.doi.org/ 10.1016/i.proci.2006.07.092.

[22] Mikulčić H, Vujanović M, Fidaros DK, Priesching P, Minić I, Tatschl R, et al. The application of CFD modelling to support the reduction of $\mathrm{CO}_{2}$ emissions in cement industry. Energy 2012:45:464-73. http://dx.doi.org/10.1016/ i.energy.2012.04.030.

[23] Iyer VA, Abraham J. Penetration and dispersion of transient gas jets and sprays. Combust Sci Technol 1997;130:315-34.

[24] Hallmann M, Scheurlen M, Wittig S. Computation of turbulent evaporating sprays: Eulerian versus Lagrangian approach. J Eng Gas Turbines Power 1995;117:112. http://dx.doi.org/10.1115/1.2812758.

[25] Durst F, Miloievic D, Schönung B. Eulerian and Lagrangian predictions of particulate two-phase flows: a numerical study. Appl Math Model 1984;8:101-15. http://dx.doi.org/10.1016/0307-904X(84)90062-3.

[26] Baleta J, Vujanović M, Pachler K, Duić N. Numerical modeling of urea water based selective catalytic reduction for mitigation of $\mathrm{NO}_{x}$ from transport sector. J Clean Prod 2014. http://dx.doi.org/10.1016/i.jclepro.2014.06.042.

[27] Harlow FH, Amsden AA. Numerical calculation of multiphase fluid flow. I Comput Phys 1975;17:19-52. http://dx.doi.org/10.1016/0021-9991(75) 90061-3.

[28] Iver VA, Abraham J, Magi V. Exploring injected droplet size effects on steady liquid penetration in a diesel spray with a two-fluid model. Int J Heat Mass Transf 2002;45:519-31. http://dx.doi.org/10.1016/S0017-9310(01)00168-5.

[29] Issa R, Oliveira P. Numerical prediction of phase separation in two-phase flow through T-junctions. Comput Fluids 1994;23:347-72. http://dx.doi.org/

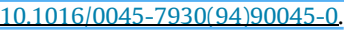


[30] Behzadi A, Issa RI, Rusche H. Modelling of dispersed bubble and droplet flow at high phase fractions. Chem Eng Sci 2004;59:759-70. http://dx.doi.org/ 10.1016/i.ces.2003.11.018.

[31] Gosman AD, Lekakou C, Politis S, Issa RI, Looney MK. Multidimensional modeling of turbulent two-phase flows in stirred vessels. AIChE J 1992;38:1946-56. http://dx.doi.org/10.1002/aic.690381210.

[32] Riber E, Moreau M, Simonin O, Cuenot B. Development of Euler-Euler LES method for gas-particle turbulent jet flow. In: Proceedings of ASME Fluids Engineering Division Summer Meeting; 2006. p. 1663-1672.

[33] Drew DA, Passman SL. Theory of multicomponent fluids; 1998.

[34] FIRE v2013 Manuals, 2013, Graz, Austria, AVL LIST GmbH; 2013.

[35] Hanjalić K, Popovac M, Hadžiabdić M. A robust near-wall elliptic-relaxation eddy-viscosity turbulence model for CFD. Int J Heat Fluid Flow 2004;25:1047-51. http://dx.doi.org/10.1016/j.ijheatfluidflow.2004.07.005.

[36] Abramzon B, Sirignano WA. Droplet vaporization model for spray combustion calculations. Int J Heat Mass Transf 1989;32:1605-18. http://dx.doi.org/ 10.1016/0017-9310(89)90043-4.

[37] Bianchi GM, Pelloni P. Modeling the diesel fuel spray breakup by using a hybrid model; 1999. http://dx.doi.org/ 10.4271/1999-01-0226.
[38] Reitz RD. Modeling atomization processes in high-pressure vaporizing sprays, Atomiz Spray Technol 1987;3:309-37.

[39] Petranović Z, Vujanović M, Duić N. Towards a more sustainable transport sector by numerically simulating fuel spray and pollutant formation in diesel engines. J Clean Prod 2014. http://dx.doi.org/10.1016/i.jclepro.2014. 09.004.

[40] von Berg E, Edelbauer W, Alajbegovic A, Tatschl R. Coupled calculation of cavitating nozzle flow, primary diesel fuel break-up and spray formation with an Eulerian multi-fluid-model. J Eng Gas Turbines Power 2004;127(4): 897-908. http://dx.doi.org/10.1115/1.1914803.

[41] O'Rourke PJ. Statistical properties and numerical implementation of a model for droplet dispersion in a turbulent gas. J Comput Phys 1989;83:345-60. http://dx.doi.org/10.1016/0021-9991(89)90123-X.

[42] Petranović Z, Edelbauer, Vujanović M, Duić N. Multiphase the O’Rourke droplet collision model for the Euler-Eulerian framework. In: Proc. 26th European Conference on Liquid Atomisation and Spray Systems (ILASS-Europe '14); 2014.

[43] Lopez de Bertodano MA. Two fluid model for two-phase turbulent jets. Nucl Eng Des 1998;179:65-74. http://dx.doi.org/10.1016/S0029-5493(97)00244-6. 